\title{
Asymptotic Properties of the Nuclear Matter
}

\section{A.I.Malakhov}

Joint Institute for Nuclear Research

Dubna, Moscow reg., Russia

E-mail: malakhovelhe.jinr.ru

Abstract

On the basis of the approach offered by A.M.Baldin and A.I.Malakhov, predictions of the yield ratio of the antiparticles to particles in the heavy ion collisions from low energy to asymptotically high energy are made. In this article we will give a comparison of our predictions made many years ago with the latest experimental data. There is a good agreement between predictions and experimental data up to LHC energy.

XXI International Baldin Seminar on High Energy Physics Problems

September 10-15, 2012

JINR, Dubna, Russia 


\section{Introduction}

About 15 years ago together with A.M.Baldin we published an article which predicted the particle yield as a result of nuclear interactions at high energy [1]. At present the corresponding experimental data have started to appear up to the LHC energy. In this talk we will give a comparison of our predictions made many years ago with the latest experimental data.

\section{Asymptotic Properties of the Nuclear Matter}

The experimentally observable quantities which characterize multi-quark processes are the cross sections of multiple particle production in relativistic nuclear collisions:

$$
\mathrm{I}+\mathrm{II} \rightarrow 1+2+3+\ldots
$$

In reaction (1) figures I and II show colliding nucleus, and by figures 1, 2, 3... secondary particles are designated. If more than one nucleon of nucleus I takes part in the interaction it is cumulative effect. The value $\mathrm{N}_{I}=\lambda \cdot \mathrm{A}_{\mathrm{I}}$, the effective number of nucleons inside nucleus I participating in the collisions, is called the cumulative number $\left(0 \leq \lambda \leq 1, \mathrm{~A}_{\mathrm{I}}\right.$ is the atomic number of the nucleus $\left.\mathrm{I}\right)$. For the cumulative region $\mathrm{N}_{\mathrm{I}}$ is more then unit $\left(\mathrm{N}_{I}>1\right)$. A double cumulative effect is also possible, when $\mathrm{N}_{\text {II }}$ is more than unit $\left(\mathrm{N}_{\mathrm{II}}>1\right)$.

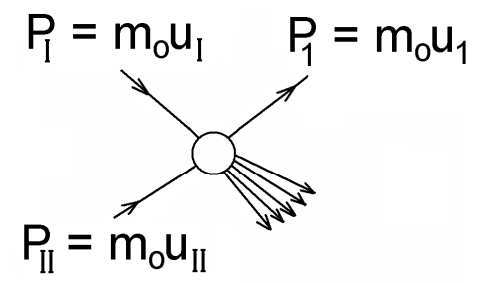

Fig.1. Interaction of two nuclei. $\mathrm{P}_{\mathrm{I}}$ is 4-momentum of nucleus $\mathrm{I}$; $\mathrm{P}_{\mathrm{II}}$ is 4-momentum of nucleus II, and $\mathrm{P}_{1}$ is 4-momentum secondary particle 1 ; $\mathrm{u}_{\mathrm{I}}, \mathrm{u}_{\mathrm{II}}, \mathrm{u}_{1}$ are 4-vilocytis of nuclei I, II and particle $1 ; \mathrm{m}_{0}$ is nucleon mass.

For the process (1), if we observe only one secondary particle (Fig.1), it is possible to write the conservation law of the 4-momentum in the following form:

$$
\left(\mathrm{N}_{\mathrm{I}} \cdot \mathrm{P}_{\mathrm{I}}+\mathrm{N}_{\mathrm{II}} \cdot \mathrm{P}_{\mathrm{II}}-\mathrm{P}_{1}\right)^{2}=\left(\mathrm{N}_{\mathrm{I}} \cdot \mathrm{m}_{0}+\mathrm{N}_{\mathrm{II}} \cdot \mathrm{m}_{0}+\Delta\right)^{2}
$$


Here $\Delta$ is the mass of the particle providing conservation of the baryon number, strangeness, and other quantum numbers. For example, if particle 1 is $\pi$ meson than $\Delta=0$.

Using the principle of symmetries, in particular, symmetries of the solutions, allowed to introduce the self-similarity parameter of $\Pi$ for the nuclear interactions [2,3]:

$$
\Pi=\min \left\{1 / 2\left[\left(\mathrm{u}_{\mathrm{I}} \cdot \mathrm{N}_{\mathrm{I}}+\mathrm{u}_{\mathrm{II}} \cdot \mathrm{N}_{\mathrm{II}}\right)^{2}\right]^{1 / 2}\right\},
$$

where $\mathrm{N}_{I}$ and $\mathrm{N}_{\text {II }}$ are cumulative numbers for nuclei I and II, and $\mathrm{u}_{\mathrm{I}}$ and $\mathrm{u}_{\mathrm{II}}$ are 4velocities of these nuclei.

In this case the invariant cross-sections of the output inclusive particles of different types at nuclear interactions with atomic numbers $A_{I}$ and $A_{I I}$, are described by universal dependence in a broad energy range and different atomic numbers of the colliding nuclei:

$$
E \cdot d^{3} \sigma / \mathrm{dp}^{3}=\mathrm{C}_{1} \cdot \mathrm{A}_{\mathrm{I}}^{\alpha\left(\mathrm{N}_{\mathrm{I}}\right)} \cdot \mathrm{A}_{\mathrm{II}}^{\alpha\left(\mathrm{N}_{\mathrm{II}}\right)} \cdot \exp \left(-\Pi / \mathrm{C}_{2}\right),
$$

where $\alpha\left(\mathrm{N}_{\mathrm{I}}\right)=1 / 3+\mathrm{N}_{\mathrm{I}} / 3, \quad \alpha\left(\mathrm{N}_{\mathrm{II}}\right)=1 / 3+\mathrm{N}_{\mathrm{II}} / 3$,

$\mathrm{C}_{1}=1.9 \cdot 10^{4} \mathrm{mb} \cdot \mathrm{GeV}^{-2} \cdot \mathrm{c}^{3} \cdot \mathrm{st}^{-1}$ и $\mathrm{C}_{2}=0.125 \pm 0.002$.

In the central rapidity region it is possible to find the analytical expression for $\Pi$ [1]. For this case $\mathrm{N}_{\mathrm{I}}$ and $\mathrm{N}_{\text {II }}$ are equal each other $\mathrm{N}_{\mathrm{I}}=\mathrm{N}_{\mathrm{II}}=\mathrm{N}$.

$$
\mathrm{N}=\left[1+\left(1+\Phi_{\delta} / \Phi^{2}\right)^{1 / 2}\right] \cdot \Phi
$$

where

$$
\begin{aligned}
& \Phi=2 \mathrm{~m}_{0} \cdot\left(\mathrm{m}_{\mathrm{T}} \operatorname{ch} \mathrm{Y}+\Delta\right) / \mathrm{sh}^{2} \mathrm{Y} \\
& \Phi_{\delta}=\left(\Delta^{2}-\mathrm{m}_{1}^{2}\right) /\left(4 \mathrm{~m}_{0}^{2} \cdot \mathrm{sh}^{2} \mathrm{Y}\right) .
\end{aligned}
$$

Here $\mathrm{m}_{1 \mathrm{~T}}$ is transverse mass of the particle $1, \mathrm{~m}_{1 \mathrm{~T}}=\left(\mathrm{m}_{1}^{2}+\mathrm{p}_{\mathrm{T}}^{2}\right)^{1 / 2}$

and than

$$
\Pi=\mathrm{N} \cdot \operatorname{ch} \mathrm{Y}
$$

From equation (4) it follows that the cross sections ratio (R) of process 1 to process 2 is as follows:

$$
\mathrm{R}=\exp \left[-\left(\Pi_{1}-\Pi_{2}\right) / \mathrm{C}_{2}\right]
$$


For baryons we have

$$
\Pi_{\mathrm{b}}=\left(\mathrm{m}_{1 \mathrm{~T}} \cdot \mathrm{chY}-\mathrm{m}_{1}\right) \cdot \operatorname{ch} \mathrm{Y} /\left(\mathrm{m}_{0} \cdot \mathrm{sh}^{2} \mathrm{Y}\right)
$$

and for antibaryons

$$
\Pi_{\mathrm{a}}=\left(\mathrm{m}_{1 \mathrm{~T}} \cdot \mathrm{chY}+\mathrm{m}_{1}\right) \cdot \operatorname{ch} \mathrm{Y} /\left(\mathrm{m}_{0} \cdot \mathrm{sh}^{2} \mathrm{Y}\right)
$$

The difference $\Pi_{\mathrm{b}}-\Pi_{\mathrm{a}}$ will not depend from $\mathrm{m}_{1 \mathrm{~T}}$ :

$$
\Pi_{\mathrm{b}}-\Pi_{\mathrm{a}}=-2 \mathrm{~m}_{1} \cdot \operatorname{chY} /\left(\mathrm{m}_{0} \cdot \operatorname{sh}^{2} \mathrm{Y}\right)
$$

The results of calculations for the ratio of antiproton to proton cross sections using (12) and (9) are presented in Fig.2 together with experimental data including the latest data at the LHC [4-6]. It is possible to see a very good agreement our calculations with experimental data in wide energy range.

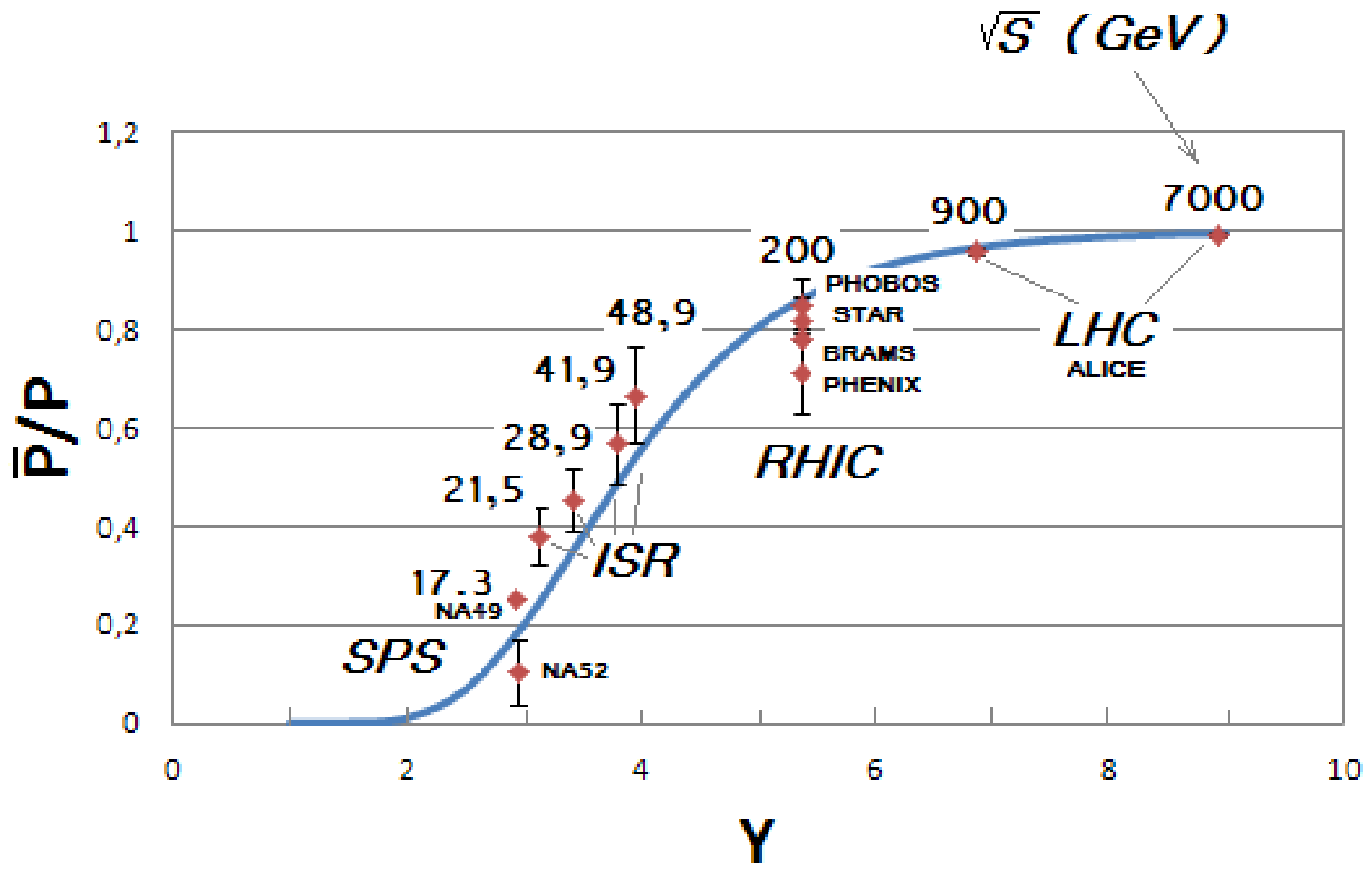

Fig.2. Dependence of the antiproton to proton cross section ratios on the rapidity and energy of interacting nuclei. 
The same calculations can be made for the antideuteron to deuteron and antihelium-3 to helium-3 yield ratios (Fig.3). However, there are poor experimental data obtained by the present moment.

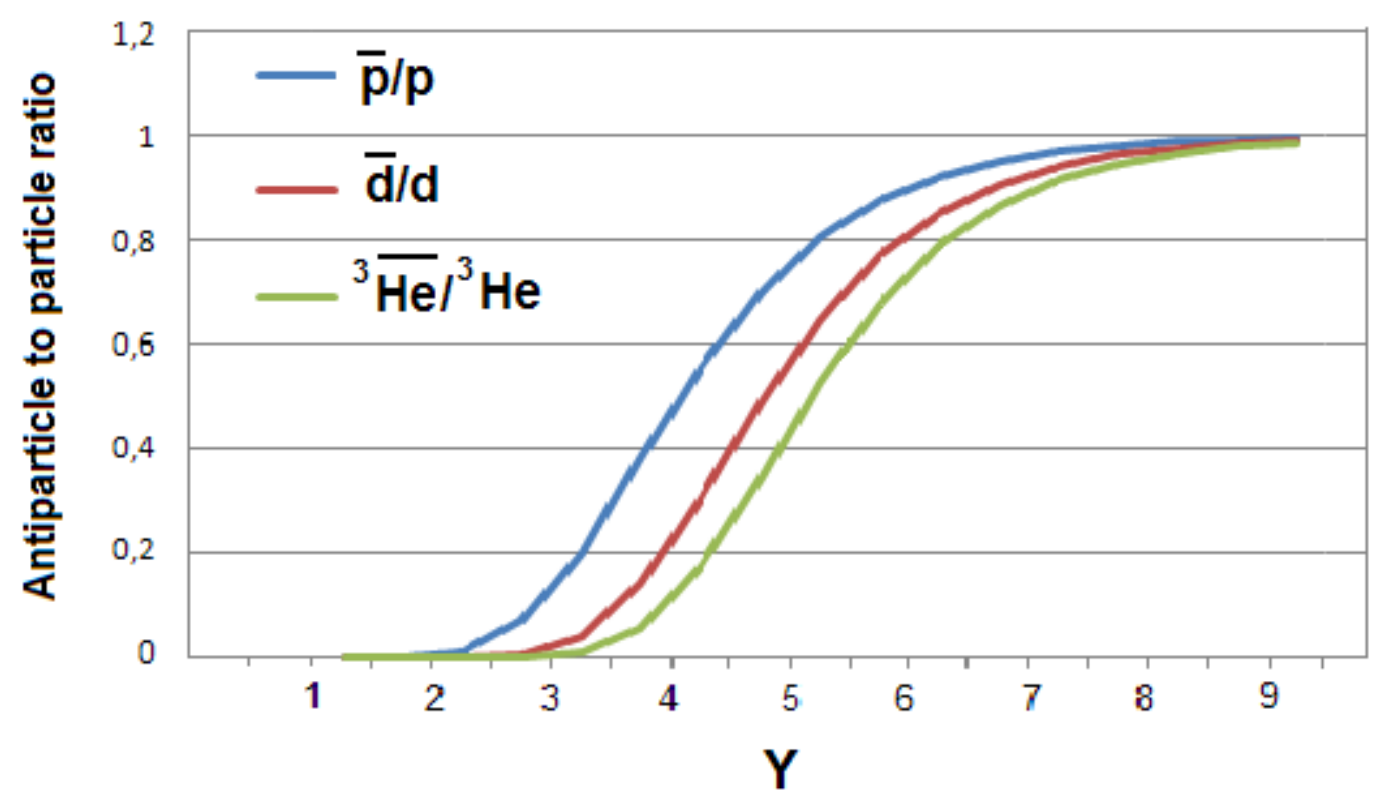

Fig.3. The results of calculations for the antiproton to proton, antideuteron to deuteron and antihelium-3 to helium-3 yield ratios.

\section{Conclusion}

The description of the nuclear interactions in the four-velocity space and introduction of the self-similarity parameter allow one to predict the cross section ratios of secondary antiprotons to protons in a wide energy range from several $\mathrm{GeV}$ to the $\mathrm{LHC}$ energy with a good accuracy.

\section{References}

[1] Baldin A.M., Malakhov A.I. JINR Rapid Communications, No.1(87)-98, 1998, pp.5-12.

[2] A.M.Baldin, A.A.Baldin. Phys. Particles and Nuclei, v.29, No.3 (1988) p.232.

[3] A.M.Baldin, A.I.Malakhov, and A.N.Sissakian. Physics of Particles and Nuclei, Vol.32. Suppl. 1, 2001, pp.S4-S30.

[4] R. Klingenberg et al. Nuclear Physics A610 (1996) 306c-316c.

[5] A. Tawfik. Nuclear Physics A 859 (2011) 63-72.

[6] http://hepdata.cedar.ac.uk/view/p7907. 